\title{
A New Three-Phase Three-Winding Transformer Electromagnetic Transient Model
}

\author{
Biao Wang ${ }^{\mathrm{a} *}$, Zhenghong Wang ${ }^{\mathrm{b}}$ \\ a Sichuan Electric Power Research Institute, Chengdu 610072, China \\ ${ }^{b}$ Southwest Petroleum University, Chengdu 610500, China
}

\begin{abstract}
This paper firstly points out a defect of the damping trapezoidal method in electromagnetic transient simulation algorithm. The backward Euler method is recommended to use. Then, a new three-phase three-winding transformer model which could consider ratio and connection group feature is put forward. Compared with PSCAD, it is obtained the proposed model is accurate and reliable.
\end{abstract}

Keywords: Electromagnetic transient, three-phase three-winding transformer, backward Euler method

\section{Introduction}

In view of the implicit trapezoidal method with considerable accuracy and good numerical stability, the electromagnetic transient program mostly uses this method. But when the grid topology changes, nonstate variables abrupt at same time, the implicit trapezoidal method calculates the equivalent current source of the abrupt time which is based on the abrupt non-state variable's value; this will result in numerical oscillation [1,2]. Obviously this oscillation is unreasonable. There are always two ways to eliminate this numerical oscillation which are backward Euler method [3-5] and the damping trapezoidal method [6]. The former is that the implicit trapezoidal method has been using when the network topology remains unchanged; and the backward Euler method is used when the network changes, then cut back to the implicit trapezoidal method. As this method avoids the abrupt non-state variable values, and only uses non-abrupt state variable's value, it could solve the problem of numerical oscillation, but its programming is slightly more complex. The damping trapezoidal method takes the numerical calculation by mixing the implicit trapezoidal method and backward Euler method together from start to end, which is regardless of the grid topology whether changed, so it is easy to program.

As to three-phase three-winding transformer model, paper [2] considered the three-winding transformer magnetizing slip alone, so it can not consider the transformer connection group. The transformer model of paper [7] is based on the controlled current source model which could consider the connection group feature and the ratio. This paper presents a new kind three-phase three-winding transformer electromagnetic transient model which takes into account the ratio and connection group feature. The transformer is taken as a coupling circuit of multi-slip series resistance and inductance. The connection group feature is achieved by the relationship of transformer's winding connection, and the ratio is simulated by the matrix of relationship of winding voltage/current and terminal voltage/current.

This paper firstly points out a serious defect of damping trapezoidal method in the electromagnetic transient simulation algorithm, and the backward Euler method is recommended. Then a new three-phase three-winding transformer model is proposed which could consider the ratio and connection group feature.

\footnotetext{
* Manuscript received June 15, 2012; revised July 20, 2012.

Corresponding author. Tel.: +86-13880914657; E-mail address: wblk-557@163.com.
} 


\section{A Default of Damping Trapezoidal Method}

The damping trapezoidal method to some extent not only maintains the advantages of implicit trapezoidal method which has high accuracy and numerical stability, but also could eliminate numerical oscillation by a certain percentage of the backward Euler method which has the damping effect, and it is easy to programming. While this method will bring certain calculation error, paper [9] proposed amendments to this method. And this paper finds another major flaw of the damping trapezoidal method, that is the current nearing a fault will numerical oscillate a certain time before transiting to the stable fault condition, and this current value of numerical oscillation may be bigger than the maximum current of the stable fault condition. It is obviously unreasonable.

Figure 1 is the electrical diagram of $220 \mathrm{kV}$ Mianyangdong intelligent substation bus I. A symmetrical three-phase instantaneous fault happens at the line L2203 and occurs at the $0.2 \mathrm{~s}$, disappears at the 0.32s. The voltage of bus I simulated by the damping trapezoidal method is shown in Figure 2 (a), and its amplified picture is shown in Figure 2 (b). It can be seen that the voltage of bus I has to numerical oscillate a period of time.

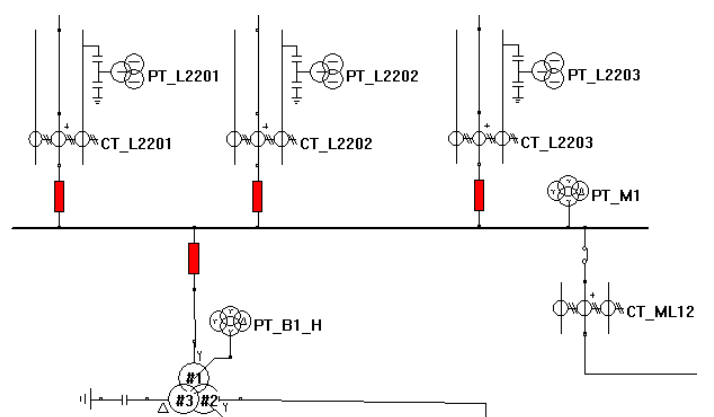

Fig.1 The electrical diagram of $220 \mathrm{kV}$ Mianyangdong intelligent substation's bus I

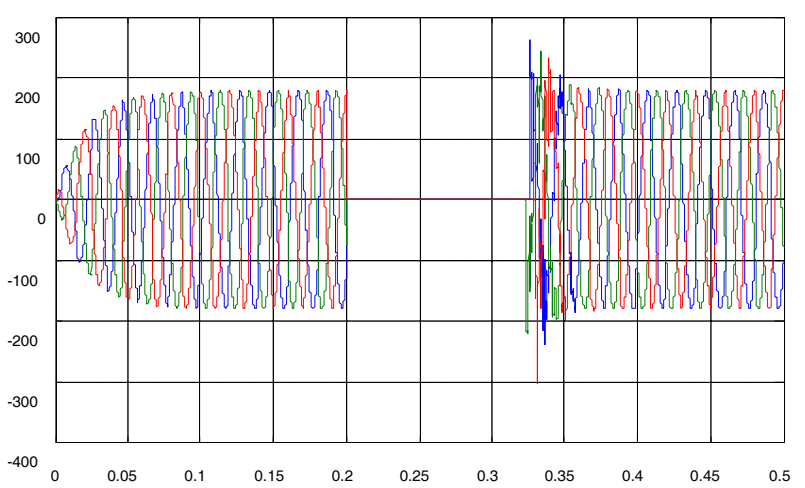

(a)

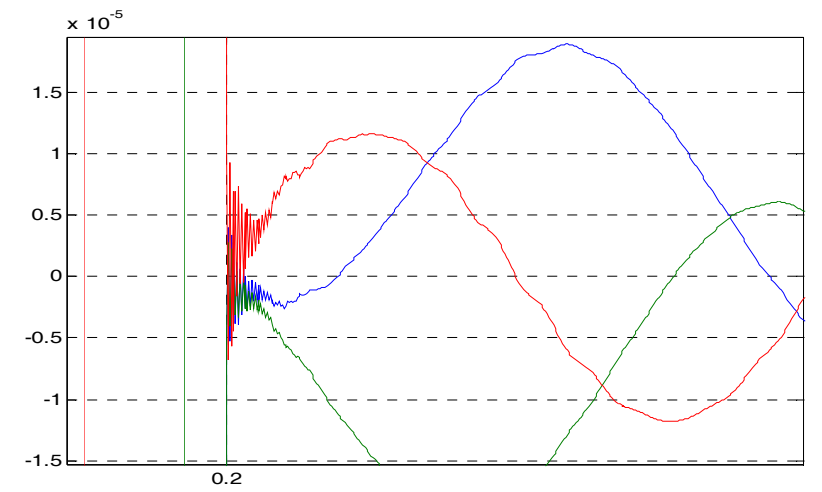

(b)

Fig.2 (a) The voltage curve of bus I simulated by damping trapezoidal method (b) The amplified voltage curve of bus I simulated by damping trapezoidal method 
Meanwhile, the simulation curve of current transformer CT_L2203's current by damping trapezoidal method is shown in Figure 3 (a). It can be seen that the CT_L2203's current has to numerical oscillate a period of time when the fault happened. The numerical oscillate current is bigger than the maximum fault current, it is obviously incorrect.

If the fault above is simulated by the backward Euler method, then the CT_L2203's current is shown in the Figure 3(b).We can see that the backward Euler method could eliminate numerical oscillation successfully, and does not has the default of damping trapezoidal method, so the widely used backward Euler method is advised to apply to eliminate numerical oscillations.

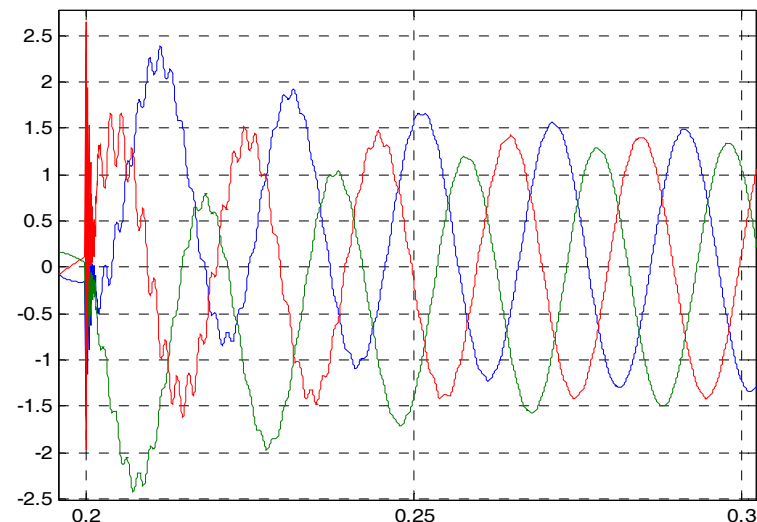

(a)

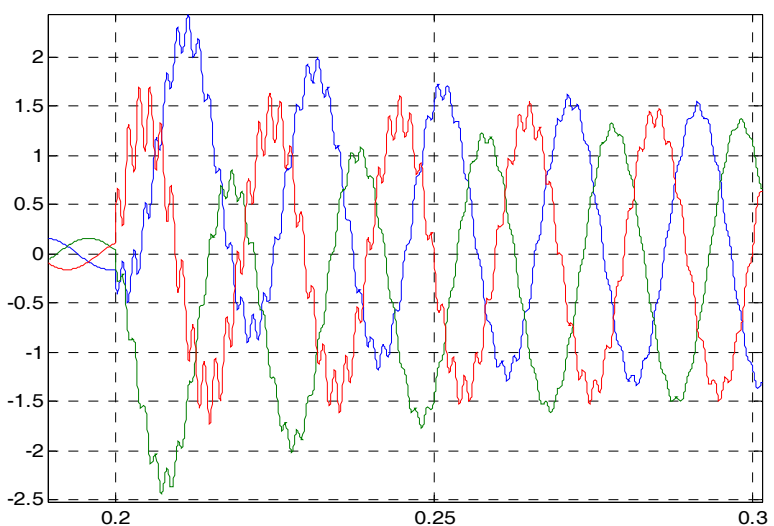

(b)

Fig.3 (a) The current curve of CT_L2203 simulated by damping trapezoidal method (b) The current curve of CT_L2203 simulated by backward Euler method

\section{A New Three-Phase Three-Winding Transformer Model}

The following describes a new three-phase three-winding transformer electromagnetic transient model which could take into account the ratio and connection group feature. While without considering the ratio, the actual circuit diagram of phase A of three-winding transformer is shown in Figure 4(a). The transient equivalent calculation circuit diagram of phase A winding is shown in Figure 4(b).Note: each winding has been converted to the side of winding first.

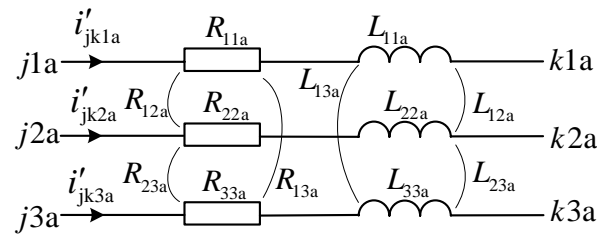

(a)

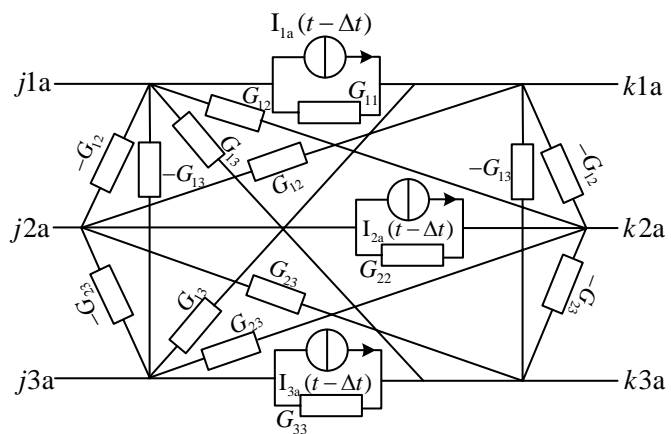

(b)

Fig.4 (a)The actual circuit diagram of three-winding transformer's phase A (b)The transient equivalent diagram of three-winding transformer's phase A

Among them, the $\mathrm{j}$ side is the port side of the transformer winding, and $\mathrm{k}$-side is the neutral point of the winding side. The voltage equation of phase $\mathrm{A}$ is

$$
\boldsymbol{L} \frac{\mathrm{d} \dot{i}_{j k a}^{\prime}(t)}{\mathrm{d} t}+\boldsymbol{R} \boldsymbol{i}_{j k a}^{\prime}(t)=\boldsymbol{u}_{j a}^{\prime}(t)-\boldsymbol{u}_{k a}^{\prime}(t)
$$




$$
\left.\begin{array}{l}
i_{j k a}^{\prime}(t)=\left[i_{j k 1 a}^{\prime}(t), i_{j k 2 a}^{\prime}(t), i_{j k 3 a}^{\prime}(t)\right]^{T} \\
\boldsymbol{u}_{j a}^{\prime}(t)=\left[u_{j 1 a}^{\prime}(t), u_{j 2 a}^{\prime}(t), u_{j 3 a}^{\prime}(t)\right]^{T} \\
\boldsymbol{u}_{k a}^{\prime}(t)=\left[u_{k 1 a}^{\prime}(t), u_{k 2 a}^{\prime}(t), u_{k 3 a}^{\prime}(t)\right]^{T}
\end{array}\right\} \quad \boldsymbol{R}=\left[\begin{array}{lll}
R_{11} & R_{12} & R_{13} \\
R_{21} & R_{22} & R_{23} \\
R_{31} & R_{32} & R_{33}
\end{array}\right]^{T} \quad \boldsymbol{L}=\left[\begin{array}{lll}
L_{11} & L_{12} & L_{13} \\
L_{21} & L_{22} & L_{23} \\
L_{31} & L_{32} & L_{33}
\end{array}\right]^{T}
$$

Among them, the $\boldsymbol{R}$ and $\boldsymbol{L}$ are respectively the resistance and reactance matrix of the phase A winding, which could be got by the winding leakage impedance and excitation impedance. $\boldsymbol{i}_{j k a}^{\prime}$ is the current vector of phase A winding, $\boldsymbol{u}_{j a}^{\prime}$ and $\boldsymbol{u}_{k a}^{\prime}$ are the $\mathrm{j}$ and $\mathrm{k}$ end of the voltage vector of phase A winding.

The impedance branches are transferred to the equivalent current source and a pure resistance by the implicit trapezoidal method. For step $t-\Delta t$ to t, the formula (1) derivate respectively to differential equations, the equivalent current source and the recursive formula by the implicit trapezoidal rule and backward Euler method, shown as formula (3) and (4).

$$
\begin{aligned}
& \boldsymbol{i}_{j k a}^{\prime}(t)=\boldsymbol{G}_{a}\left[\boldsymbol{u}_{j a}^{\prime}(t)-\boldsymbol{u}_{k a}^{\prime}(t)\right]+\boldsymbol{I}_{a}(t-\Delta t) \\
& \boldsymbol{I}_{a}(t-\Delta t)=\boldsymbol{I}_{\mathrm{coa}} \boldsymbol{i}_{j k a}^{\prime}(t-\Delta t)+\boldsymbol{G}_{a}\left[\boldsymbol{u}_{j a}^{\prime}(t-\Delta t)-\boldsymbol{u}_{k a}^{\prime}(t-\Delta t)\right] \\
& \left.=\boldsymbol{I}_{c o a} \boldsymbol{I}_{a}(t-2 \Delta t)+\boldsymbol{U}_{c o a}\left[\boldsymbol{u}_{j a}^{\prime}(t-\Delta t)-\boldsymbol{u}_{k a}^{\prime}(t-\Delta t)\right]\right) \\
& \mathbf{i}_{j k a o}^{\prime}(t)=\boldsymbol{G}_{a}\left[\boldsymbol{u}_{j a}^{\prime}(t)-\boldsymbol{u}_{k a}^{\prime}(t)\right]+\boldsymbol{I}_{a o}(t-\Delta t) \\
& \boldsymbol{I}_{a o}(t-\Delta t)=\boldsymbol{G}_{a} \frac{2 \boldsymbol{L}}{\Delta t} \boldsymbol{i}_{j k a o}^{\prime}(t-\Delta t) \\
& \left.=\boldsymbol{G}_{a} \frac{2 \boldsymbol{L}}{\Delta t} \boldsymbol{I}_{a o}(t-2 \Delta t)+\boldsymbol{G}_{a} \frac{2 \boldsymbol{L}}{\Delta t} \boldsymbol{G}_{a}\left[\boldsymbol{u}_{j a}^{\prime}(t-\Delta t)-\boldsymbol{u}_{k a}^{\prime}(t-\Delta t)\right]\right]
\end{aligned}
$$

where $\boldsymbol{G}_{a}=[2 \boldsymbol{L} / \Delta t+\boldsymbol{R}]^{-1}, \boldsymbol{I}_{\text {со }}=\left[\boldsymbol{E}-2 \boldsymbol{G}_{a} \boldsymbol{R}\right]$ and $\boldsymbol{U}_{\text {со }}=2\left[\boldsymbol{G}_{a}-\boldsymbol{G}_{a} \boldsymbol{R} \boldsymbol{G}_{a}\right]$

Among them, $\boldsymbol{E}$ is unit matrix, $\boldsymbol{G}_{a}$ is the conductance matrix of corresponding impedance branch by the implicit trapezoidal method, $\boldsymbol{I}_{\text {соа }}$ is coefficient matrix of current source by the implicit trapezoidal method, $\boldsymbol{U}_{\text {со }}$ is coefficient matrix of voltage by the implicit trapezoidal method.

As to the transformer ratio, it is simulated by the relationship matrix of the winding voltage/current and terminal voltage/current. While the transformer parameters have been converted to winding first side, the voltage and current relationship matrix is

$$
\left.\left.\begin{array}{l}
u_{j 1 a}-u_{k 1 a}=u_{j 1 a}^{\prime}-u_{k 1 a}^{\prime} \\
u_{j 2 a}-u_{k 2 a}=\left(u_{j 2 a}^{\prime}-u_{k 2 a}^{\prime}\right) / k_{12} \\
u_{j 3 a}-u_{k 3 a}=\left(u_{j 3 a}^{\prime}-u_{k 3 a}^{\prime}\right) / k_{13}
\end{array}\right\}, \quad \begin{array}{l}
i_{i j 1 a}=i_{i j 1 a}^{\prime} \\
i_{i j 2 a}=k_{12} i_{i j 2 a}^{\prime} \\
i_{i j 3 a}=k_{13} i_{i j 3 a}^{\prime}
\end{array}\right\}
$$

Among them, $k_{12}$ is the ratio of winding first's voltage to winding second's, $k_{13}$ is the ratio of winding first's voltage to winding third's, $\boldsymbol{u}_{j a}$ and $\boldsymbol{u}_{k a}$ are respectively the voltage vector of transformer phase A's the port side and the neutral point side after considering the ratio. The transformer model formula considering the ratio could be gotten by the equation (5) inserted into the equation (3), and it is as follows: 


$$
\begin{aligned}
& \boldsymbol{i}_{j k a}(t)=\boldsymbol{G}_{T}\left[\boldsymbol{u}_{j a}(t)-\boldsymbol{u}_{k a}(t)\right]+\boldsymbol{I}_{T a}(t-\Delta t) \\
& \begin{aligned}
& \boldsymbol{I}_{T a}(t-\Delta t)=\boldsymbol{I}_{T c o a} \boldsymbol{i}_{j k a}(t-\Delta t)+\boldsymbol{G}_{T a}\left[\boldsymbol{u}_{j a}(t-\Delta t)-\boldsymbol{u}_{k a}(t-\Delta t)\right] \\
&= \boldsymbol{I}_{T c o a} \boldsymbol{I}_{T a}(t-2 \Delta t)+\boldsymbol{U}_{T c o a}\left[\boldsymbol{u}_{j a}(t-\Delta t)-\boldsymbol{u}_{k a}(t-\Delta t)\right]
\end{aligned} \\
& \boldsymbol{G}_{T a}=\boldsymbol{T} \boldsymbol{G}_{a} \boldsymbol{T}, \quad \boldsymbol{I}_{T c o a}=\boldsymbol{T}\left(\boldsymbol{E}-2 \boldsymbol{G}_{a} \boldsymbol{R}\right) \boldsymbol{T}^{-1} \\
& \boldsymbol{U}_{T c o a}=2 \boldsymbol{T}\left[\boldsymbol{G}_{a}-\boldsymbol{G}_{a} \boldsymbol{R} \boldsymbol{G}_{a}\right] \boldsymbol{T}, \quad \boldsymbol{T}=\operatorname{diag}\left(1, k_{12}, k_{13}\right)
\end{aligned}
$$

As it is easy to get the backward Euler method formula of transformer which is considered the ratio, by substituting equation (5) into equation (4) accordingly, this is omitted.

So it is easy to achieve the simulation of the transformer ratio. The model and formula of phase B and $\mathrm{C}$ are similar to phase A.

As to the simulation of transformer's connection group feature, it is easy to get the transformer conductance matrix and the relationship of node injection current and the equivalent current source. According to the transformer $\mathrm{ABC}$ three-phase transient equivalent circuit which is based on transformer's connection way, it could make transient calculation gradually. The reason why transformer's connection way is not simulated similar to the ratio matrix is that the matrix of connection way is irreversible when the connection way has a triangle way.

The $220 \mathrm{kV}$ Mianyangdong intelligent substation is simulated by the PSCAD and this paper's procedure. A fault happens on the line of Mianyangdong - Baozhusi in 0.2s, and disappears in 0.32s. The voltage curve of bus I on the high voltage side are showed in Figure 5(a) and Figure 5(b), and the voltage curve of the neutral point of the high voltage side are showed in Figure 6(a) and Figure 6(b). The comparison result of these four figures is showed in Table 1.

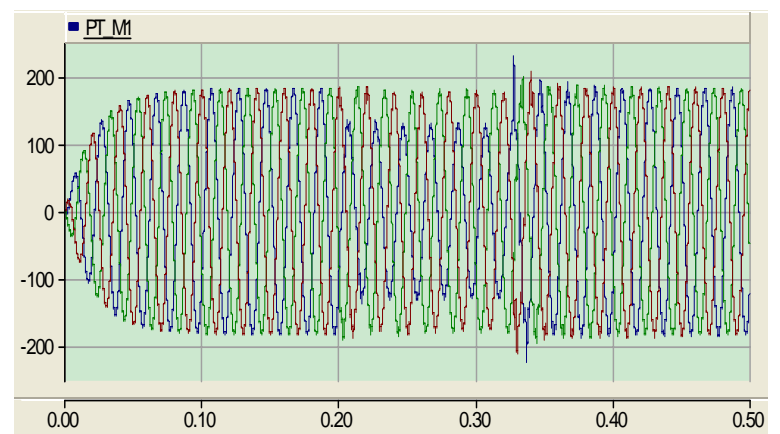

(a)

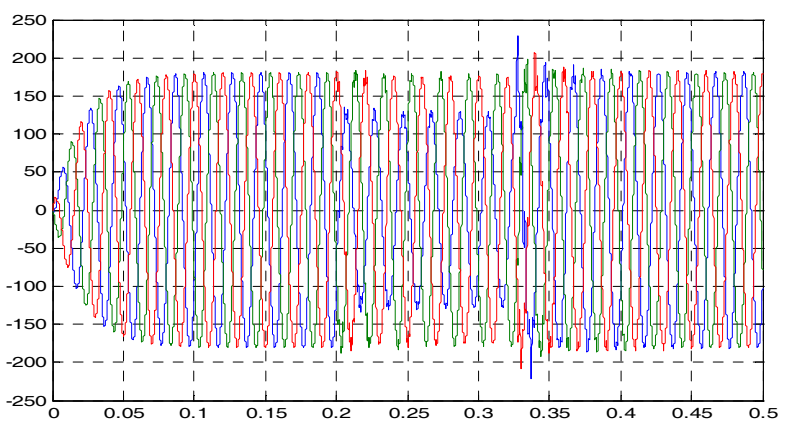

(b)

Fig.5 (a) The voltage curve of bus I simulated by PSCAD (b) The voltage curve of bus I simulated by this paper

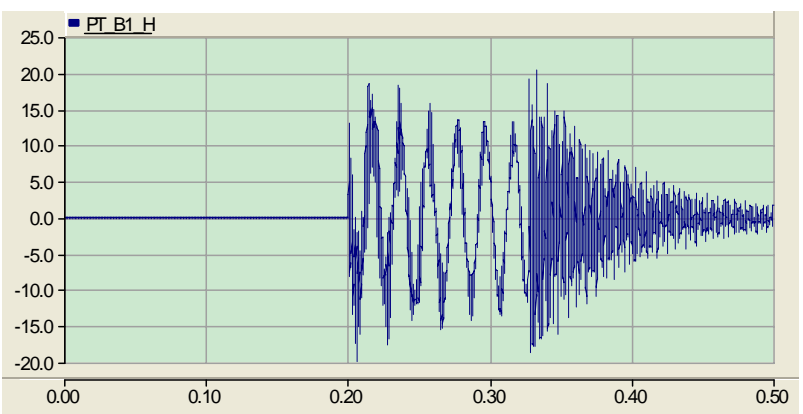

(a)

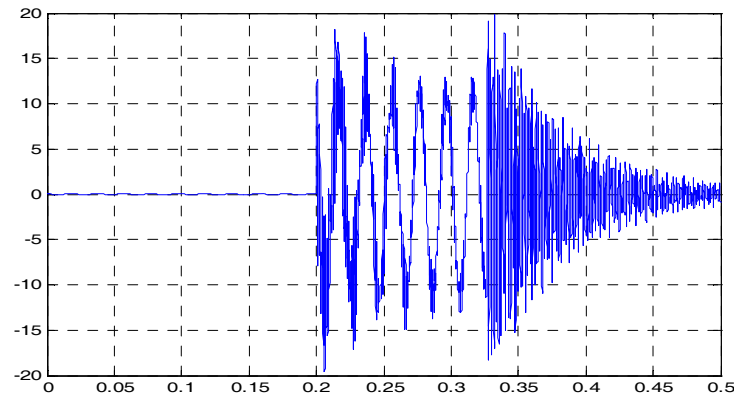

(b)

Fig.6 (a) The voltage curve of 1\# transformer's high voltage side neutral point by PSCAD (b) The voltage curve of 1\# transformer's high voltage side neutral point by this paper 
Tab. 1 The results compared by PSCAD and this paper

\begin{tabular}{c|c|c|c|c}
\hline \multicolumn{2}{c|}{ Parameter } & PSACD $(\mathrm{KV})$ & This paper(KV) & error \\
\hline \multicolumn{2}{c|}{ The transient voltage of bus I of phase A } & 228.71 & 228.31 & $-0.17 \%$ \\
\hline \multirow{2}{*}{$\begin{array}{c}\text { The maximum voltage of \#1transformer's } \\
\text { high voltage side neutral point }\end{array}$} & During fault & -19.55 & -19.80 & $1.28 \%$ \\
\cline { 2 - 5 } & After fault & 19.84 & 20.42 & $2.92 \%$ \\
\hline
\end{tabular}

Note: error $=(\mid$ this paper|-|PSCAD|)/|PSCAD|.

As showed in Table 1, the result's maximum error of the PSCAD and this paper does not exceed 3\%, it can prove that the proposed three-phase three-wind transformer model is accurate and reliable.

\section{Conclusion}

This paper points out a serious defect of the damping trapezoidal method in electromagnetic transient simulation, and proposes a new three-phase three-winding transformer model which takes into account of the characteristics of the ratio and connection group features. It's proved this model is accurate, reliable and easy to program implementation by compared results of the PSCAD and this paper.

\section{References}

[1] Lin YZ, Li JM, Zeng SH. The Theory of Power System Electromagnetic Transient Calculation. Beijing: Water Resources and Electric Power Press; 1991. (in Chinese)

[2] Xia DZ. Analysis of Power System. Beijing: China Electric Power Press; 1995.

[3] Lin JM, Marti H. Implementation of the CDA procedure in the EMTP. IEEE Trans. on Power Systems, 1990; 5(2):394-402.

[4] Zhang Y, Zhou Q. Numerical oscillation and its countermeasures in power system simulation. Journal of Shanghai Jiaotong University, 1999; 33(2):1545-1549.

[5] Liu WZ, Tang Y, Hou JX. Simulation algorithm for multi variable-step electromagnetic transient considering multiple events. Proceedings of the CSEE, 2009; 29(34):57-61.

[6] Huang JY, Chen LY, Sun DC. Power system Digital Simulation. Beijing: Water Resources and Electric Power Press; 1995.

[7] Wang QP, Dong XZ, Zhou SX. Whole transient calculation of transformer based on self-adaptive model. Automation of Electric Power Systems, 2004, 28(18):54-58.

[8] Liu YQ, Chen CY. Errors analysis and correction of damping trapezoidal integration for eliminating numerical oscillations. Proceedings of the CSEE, 2003, 23(7):57-61. 